\title{
Comment on Schosser (2018) “Incentive Systems for Risky Investment Decisions under Unknown Preferences: Ortner et al. Revisited"
}

\author{
Julia Ortner
}

Chair of Management Accounting, Department of Law and Economics, Johannes Gutenberg University Mainz, 55099 Mainz, Germany; julia.ortner@uni-mainz.de

Received: 25 October 2018; Accepted: 12 November 2018; Published: 14 November 2018

\begin{abstract}
Schosser (Games 2018, 9, 26) claims to have found an alternative solution to design appropriate performance measures than the State-Contingent Relative Benefit Cost Allocation (RBCA) introduced by Ortner et al. (Management Accounting Research 2017, 36, 43-50), which he states is simpler and more powerful. However, this note reveals that the performance measures proposed by Schosser are, in fact, a specific subset of State-Contingent Robust RBCA performance measures and thus do not represent a new solution.
\end{abstract}

Keywords: investment decision; performance measure; relative benefit cost allocation

\section{Introduction}

Ortner et al. [1] examine how to provide a manager whose time and risk preferences are both unknown with incentives to make value-maximizing investment decisions in a risky setting. They introduce the so-called State-Contingent (Robust) Relative Benefit Cost Allocation (RBCA) scheme, which leads to timewise and statewise dominant performance measures for the value-maximizing investment strategy by distributing a specific portion of the expected net present value (NPV) in every state in each period. In his note "Incentive Systems for Risky Investment Decisions under Unknown Preferences: Ortner et al. Revisited" (Games 2018, 9, 26) Schosser claims to present a simpler and more powerful solution to design appropriate performance measures than the State-Contingent RBCA scheme introduced by Ortner, Velthuis, and Wollscheid in "Incentive systems for risky investment decisions under unknown preferences" (Management Accounting Research 2017, 36, 43-50). However, I demonstrate that the "Schosser performance measures" are a subset of State-Contingent Robust RBCA performance measures.

\section{Main Setting}

Ortner et al. (2017) [1] show how to construct so-called "consistent" accrual based performance measures for risky investment decisions, i.e., providing a probably risk averse manager in charge of the investment decisions with financial incentives to implement the investment strategy that maximizes expected overall net present value (E(NPV)).

The framework of their model, which Schosser [2] refers to, is as follows: A risk-neutral owner of a firm delegates risky investment decisions to a manager, which occur in $t=0$. If the manager decides to invest, the investment requires an initial investment expenditure $I$ in $t=0$ and subsequently generates risky (or riskless) cash flows $c_{t s}\left(c_{t}\right)$ in state $s$ at times $1 \leq t \leq T$. The cash flows are represented as the product of specific factors, i.e.,

$$
c_{t s}(I)=\psi_{t s} \cdot E\left(c_{t}(I)\right)=\psi_{t s} \cdot x_{t} \cdot y(I) \text { with } E\left(\psi_{t}\right)=1 \text {, }
$$


where $\psi_{t s}$ represents the state specific variation of cash flows with respect to the expected cash flow and the expected cash flow is the product of a (period specific) temporal growth factor $x_{t}$ and a (period independent) profitability factor $y(I)$.

The manager has an information advantage over the owner. Only the manager has ex ante complete information of possible investment projects, i.e., only he knows the investment expenditure $I$, possible future periodic cash flows $c_{t s}$ in the different states and the probability of each environmental state $p_{t s}$. However, as soon as cash flows resp. the initial investment expenditure $\left(c_{t s}\right.$ resp. $\left.I\right)$ are realized, they can be observed by the owner.

To align their financial interests, the owner establishes an incentive system by designing performance measures $\pi_{t s}$ and by specifying the functional relationship between the performance measures and the variable compensation of the manager $\omega_{t}$ at each point in time $1 \leq t \leq T$ :

$$
\omega_{t}=\omega_{t}\left(\pi_{t s}\right) .
$$

Performance measures can be based on the initial investment expenditure and already realized cash flows, i.e.,

$$
\pi_{t s}=\pi_{t s}\left(I, c_{1 s}, \ldots, c_{t s}\right) .
$$

As the manager's preferences are assumed to be unknown to the owner, to ensure preference similarity, it is—as shown by Ortner et al. (2017) [1]—crucial that the performance measures always reflect a project-independent (positive) proportion of the expected NPV of the investment, i.e.,

$$
\pi_{t s}=\lambda_{t s} \cdot E(N P V(I)) \forall t, s .
$$

\section{Results: State-Contingent Robust Relative Benefit Cost Allocation (RBCA) as a Solution}

\subsection{Characteristics of the State-Contingent Robust RBCA by Ortner et al.}

Ortner et al. (2017) [1] reveal the minimal information requirement, which is necessary to enable constructing performance measures with this property. In particular, at latest in the contracting period $t$ the temporal growth factors $x_{\tau}$ of all (expected) cash flows and the realized variation factor $\psi_{t s}$ have to be known.

In a setting with performance measures that allocate both cash flows and initial investment expenditure (linearly) over time, i.e., $\pi_{t s}=\alpha_{t s} \cdot c_{t s}(I)-\hat{A}_{t s} \cdot I \equiv \hat{c}_{t s}(I)-\hat{A}_{t s} \cdot I$, Ortner et al. (2017) [1] introduce the corresponding so-called State-Contingent Robust Relative Benefit Cost Allocation (RBCA) Scheme, i.e.,

$$
\hat{A}_{t s}^{\text {State-Contingent Robust RBCA }}=\underbrace{\alpha_{t s} \cdot \psi_{t s} \cdot x_{t}}_{\equiv \hat{\psi}_{t s} \cdot \hat{x}_{t}} \cdot \frac{1}{\sum_{\tau=1}^{T} \hat{x}_{\tau} \cdot \gamma_{P}^{\tau}}=\hat{\psi}_{t s} \cdot \frac{\hat{x}_{t}}{\sum_{\tau=1}^{T} \hat{x}_{\tau} \cdot \gamma_{P}^{\tau}} .
$$

which is based on this information and constitutes a linear, state-specific cost allocation rule based on the structure of the allocated cash flows, i.e.,

$$
\hat{c}_{t s}=\alpha_{t s} \cdot \sum_{i} \underbrace{c_{t s i}}_{\psi_{t s i} \cdot x_{t i} \cdot y_{i}}=\sum_{i} \underbrace{\alpha_{t s i} \cdot c_{t s i}}_{\hat{\psi}_{t s i} \cdot \hat{x}_{t i} \cdot y_{i}}=\sum_{i} \hat{\psi}_{t s i} \cdot \hat{x}_{t i} \cdot y .
$$

If investments are exclusive, cash flow allocation has to induce identical time and risk structures to all investment projects, i.e., $\hat{\psi}_{t s i}=\hat{\psi}_{t s}$ and $\hat{x}_{t i}=\hat{x}_{t} \forall i$. 


\section{2. "Schosser Solution" as a Subset of the State-Contingent Robust RBCA Performance Measures}

Schosser [2] proposes basing performance measures directly on information about expected cash flows in terms of expected NPV (stated in Equation (2) in his note), ${ }^{1}$ i.e.,

$$
\pi_{t}=\kappa_{t} \cdot \underbrace{\left(\sum_{\tau=1}^{T} x_{\tau} \cdot y(I) \cdot \gamma_{P}^{\tau}-I\right)}_{\equiv E(N P V)} .
$$

It is important to note- as also correctly stated by Schosser [2] — that at the time of the incentive contract design expected future cash flows (resp. expected NPV) are still unknown to the owner.

Thus, this condition stated in (7) constitutes a property of consistent performance measures, which is identical to that stated in (4), rather than a possible contract for the manager.

However, as Schosser shows [2], a contract according to (7) could be written in combination with a rule determining how to derive the unknown profitability factor based on the observed cash flows and the available information in the respective period. In particular: ${ }^{2}$

$$
y(I)=f\left(c_{t s}(I), \psi_{t s}, x_{t}\right)=\frac{1}{\psi_{t s} \cdot x_{t}} \cdot c_{t s}(I)
$$

It can be noted that this rule determines a share of the realized periodic cash flows and thus can be interpreted as a specific cash flow weighting, which is both time and state specific.

To analyze the contract proposed by Schosser [2], I insert its second part according to (8) into the first according to (7) (and thus consider a single contract incorporating both contract parts proposed by Schosser [2]), i.e.,

$$
\begin{aligned}
\pi_{t} & =\kappa_{t} \cdot(\underbrace{\sum_{\tau=1}^{T} x_{\tau} \cdot y(I) \cdot \gamma_{P}^{\tau}-I}_{E(N P V)}) \text { with } y(I)=\frac{c_{t s}(I)}{\psi_{t s} \cdot x_{t}} \\
& =\kappa_{t} \cdot\left(\sum_{\tau=1}^{T} x_{\tau} \cdot \frac{c_{t s}(I)}{\psi_{t s} \cdot x_{t}} \cdot \gamma_{P}^{\tau}-I\right) \\
& =\underbrace{\left(\kappa_{t} \cdot \frac{1}{\psi_{t s} \cdot x_{t}} \cdot \sum_{\tau=1}^{T} x_{\tau} \cdot \gamma_{P}^{\tau}\right)}_{\equiv \bar{\alpha}_{t s}} \cdot c_{t s}(I)-\underbrace{\kappa_{t}}_{\equiv \hat{A}_{t s}} \cdot I .
\end{aligned}
$$

The presentation in (9) shows that it would also be possible to interpret this contract as an accrual accounting measure with a specific (linear) cash flow allocation rule $\bar{\alpha}_{t s}$ and a (linear) cost allocation rule $\kappa_{t}$. Hence, due to the allowance for cash flow allocation-unlike the procedure by Schosser [2] - these performance measures have to be compared to the State-Contingent Robust RBCA

1 Schosser [2] states that possibility of applying his solution would be specific to the risky framework in the Ortner et al. paper [1] and not apply to earlier models assuming risk neutrality and the existence of a (unknown) noise term. However, in these models, it would be sufficient to construct performance measures that reflect a positive portion of a "noisy estimate" of expected NPV (based on a noisy signal). In order to so, the "second contract" would state how to determine a noisy signal of the profitability factor (rather than the real profitability factor, which remains unknown), in particular: $\hat{y}(I)=c_{t s}(I) \cdot x_{t}^{-1}$. This signal in expectations would equal the real profitability factor, i.e., $E(\hat{y}(I))=E\left(\tilde{c}_{t s}(I)\right) \cdot x_{t}^{-1}=y$, as in expectations the error term equals zero. Due to the assumed risk neutrality of the manager in these earlier models, it would not harm the incentive effect that the real performance measure may deviate from the expected performance measures.

2 Schosser [2] also mentions this determination rule. However, when evaluating his performance measures, he does not consider that it has to be part of the contract. 
performance measures rather than to the "simple" state-contingent RBCA performance measures which only allow for a cost allocation.

To express the performance measures in terms of allocation rules (rather than portion of NPV), I insert $\kappa_{t}=\bar{\alpha}_{t s} \cdot \psi_{t s} \cdot x_{t} \cdot \frac{1}{\sum_{\tau=1}^{T} x_{\tau} \cdot \gamma_{P}^{\tau}}$ into (9), which leads to

$$
\pi_{t}=\bar{\alpha}_{t s} \cdot c_{t s}(I)-\underbrace{\bar{\alpha}_{t s} \cdot \underbrace{\psi_{t s} \cdot x_{t} \cdot \frac{1}{\sum_{\tau=1}^{T} x_{\tau} \cdot \gamma_{P}^{\tau}}}_{\text {State-Contingent RBCA }}}_{\text {State-Contingent Robust RBCA }} \cdot I
$$

with $\bar{\alpha}_{t s}=\kappa_{t} \cdot \frac{1}{\psi_{t s} \cdot x_{t}} \cdot \sum_{\tau=1}^{T} x_{\tau} \cdot \gamma_{P}^{\tau}$. In this presentation, it becomes obvious, that the cost allocation rule is exactly the State-Contingent Robust RBCA scheme introduced by Ortner et al. [1]. In contrast to the general Ortner et al. solution, in the "Schosser solution" a specific cash flow allocation rule is chosen. To conclude, the performance measures proposed by Schosser [2] are a specific subset of State-Contingent Robust RBCA performance measures and thus do not represent a new solution.

\subsection{Reply to Further Related Objections by Schosser}

It can be noted that the (implied) allocation rules are state-dependent. In his note, Schosser [2] (correctly) observes that the resulting performance measures however are not necessarily state-dependent, in the sense that its magnitude does not depend on the realized state, i.e., $\pi_{t s}=\pi_{t} \forall s$. He claims this property of the performance measure to be in contrast to the result of Ortner et al. "that appropriate performance measures necessarily have to be state-dependent" [1]. However, this is not true. Ortner et al. themselves state in Section 4.2 of their paper [1] that non-state-dependent, in other words risk-free performance measures (i.e., $\pi_{t s}=\pi_{t} \forall s$ ) can be always obtained if allowing for cash flow allocation [1]. (The need for state-depend performance measures, which is referred to by Schosser [2], only holds true in a setting not allowing for cash flow allocation.).

Schosser [2] claims his solution to be more powerful. As his solution represents a subset of the general solution by Ortner et al., this does not hold. His perception results from the fact that he compares it to the simple state-contingent RBCA scheme rather than to the State-Contingent Robust RBCA scheme. Schosser [2] considers his solution not to require a cash flow allocation and thus to be easier. However, as shown, technically his solution corresponds to a cash flow (and cost) allocation rather than solely a cost allocation. Hence, as already stated, due to (the analogy to) the consideration of cash flow allocation in the contract proposed by Schosser [2] (according to (8)), it has to be compared to the State-Contingent Robust RBCA scheme. The (State-Contingent) Robust RBCA scheme generates consistent performance measures for all types of projects rather than only for "normal projects". Thus, this critique of Schosser [2] does not apply with respect to the State-Contingent Robust RBCA performance measures.

\section{Discussion}

This note reveals that the performance measures proposed by Schosser [2] are only a specific subset of State-Contingent Robust RBCA performance measures. Hence, they do not constitute an alternative solution to the State-Contingent Robust RBCA scheme (by Ortner et al. [1]) to induce consistent incentives for (risky) investment decisions by a manager under unknown preferences. In that regard, it is important to understand that the "Schosser solution" has to be compared to the State-Contingent Robust RBCA scheme rather than the "simple" state-contingent RBCA scheme, which I explain in this note. Finally, it becomes obvious that the "Schosser solution" technically relies on exactly the same mechanism as the State-Contingent Robust RBCA performance measures. 
Funding: This research received no external funding.

Acknowledgments: I would like to thank Louis Velthuis for helpful comments.

Conflicts of Interest: The author declares no conflict of interest.

\section{References}

1. Ortner, J.; Velthuis, L.; Wollscheid, D. Incentive system design for risky investment decisions under unknown preferences. Manag. Account. Res. 2017, 36, 43-50. [CrossRef]

2. Schosser, J. Incentive Systems for Risky Investment Decisions Under Unknown Preferences: Ortner et al. Revisited. Games 2018, 9, 26. [CrossRef] 